\title{
Divides with cusps and Kirby diagrams for line arrangements
}

\author{
Sakumi Sugawara*, Masahiko Yoshinaga ${ }^{\dagger}$ \\ Dedicated to the memory of Prof. Stefan Papadima
}

July 6, 2021

\begin{abstract}
The complement of a complexified real line arrangement is an affine surface. It is classically known that such a space has a handle decomposition up to 2-handles. We will describe the handle decomposition induced from Lefschetz hyperplane section theorem for such a space. To describe the Kirby diagram, we introduce the notion of the divide with cusps which is a generalization of the divide introduced by A'Campo.
\end{abstract}

Keywords: line arrangements, divides, Kirby diagrams

MSC classes: 32S50, 32Q55, 52C35

\section{Introduction}

Let $\mathcal{A}=\left\{H_{1}, \ldots, H_{n}\right\}$ be a set of hyperplanes in a complex vector space $\mathbb{C}^{\ell}$. The complement $M(\mathcal{A})=\mathbb{C}^{\ell} \backslash \bigcup_{H \in \mathcal{A}} H$ is an important topological space that has been studied from the topological and combinatorial perspective ([OT92]). One of the most peculiar properties of $M(\mathcal{A})$ is the socalled minimality, that is, $M(\mathcal{A})$ is homotopy equivalent to a finite $\mathrm{CW}$ complex such that the number of $k$-cells is equal to the $k$-th Betti number $b_{k}(M(\mathcal{A}))$ for all $k \geq 0$ [R02, DP03] (see also [F93] for the case $\ell=2$ ). Note that the original proof did not give a precise description of attaching maps of minimal CW complexes. Later several approaches to this problem were developed for complexified real arrangements. For example, descriptions via Lefschetz hyperplane section theorem [Y07] and via discrete Morse theory [SS07] are known.

When $\ell=2$, the complement $M(\mathcal{A})$ is a complex smooth affine surface. It has been well known that such a space is a 4-manifold which has a handle decomposition by 0-, 1-, and 2handles. Such handle decompositions are completely described by Kirby diagrams ( Ak16, K89, GS99]). However, as far as the authors know, there are no results available in the literature concerning explicit handle decompositions and Kirby diagrams for $M(\mathcal{A})$. (Recently, the handle decompositions for the complement of real 1-dimensional subspaces in $\mathbb{R}^{\ell}$ were obtained [IO20].) The purpose of the present paper is to give a description of handle decompositions and Kirby diagrams of $M(\mathcal{A})$ for complexified real line arrangements.

Our approach consists of two steps. The first step is describing the handle decomposition explicitly (\$4). This part is a refinement of a previous description ([Y07, Y12]) of the attaching

${ }^{*}$ Department of Mathematics, Graduate School of Science, Hokkaido University, North 10, West 8, Kita-ku, Sapporo 060-0810, JAPAN E-mail: sugawara.sakumi.f5@elms.hokudai.ac.jp

${ }^{\dagger}$ Department of Mathematics, Faculty of Science, Hokkaido University, North 10, West 8, Kita-ku, Sapporo 060-0810, JAPAN E-mail: yoshinaga@math.sci.hokudai.ac.jp 
maps of 2-cells on 1-skeletons. We will describe the attaching of 2-handles using piecewise linear maps.

The second step is describing the Kirby diagram explicitly. For this purpose, we introduce the notion of the divide with cusps.

Simply speaking, a divide $C$ is a union of immersed curves in the 2-dimensional unit disk $D^{2}$. Although the divide was originally introduced in the context of complex plane curve singularities, it is of interest in low dimensional topology since one can associate a link $L(C)$ in $S^{3}$ to a divide $C$ ([AC98]). The notion of the divide can be considered as a "2-dimensional way" of describing certain links. There are also several generalizations of the notion of divide, e.g., oriented divides and free divides (GI02a, GI02b]), etc. (In particular, the notion of cusp in the present paper may be considered as "divisors" (in the sense of [GI02b]) equipped with half-integer signs $\pm \frac{1}{2}$.)

The divide with cusps is, roughly speaking, a generalization that allows cusps in the union of curves $C$ in the $D^{2}$. Since the tangent space is well-defined also at a cusp, one can associate a link as in the case of divides. Our main result asserts that there exists a divide with cusps such that its link represents the Kirby diagram of $M(\mathcal{A})$.

The paper is organized as follows. In $\$ 2$ we prepare piecewise linear descriptions of the 3sphere $S^{3}$ and certain circles in it which will be used later. In $\$ 3$ we define divides with cusps and associated links. In $\$ 4$ we give a piecewise linear description of handle decompositions for the complements to complexified real line arrangements. Here Kirby diagrams (the framed links consisting of boundaries of carved disks and attaching circles of 2-handles) are described in terms of piecewise linear curves introduced in the previous section. In $\$ 5$ we describe the Kirby diagram using divide with cusps.

\section{Standard and PL models for $S^{3}$}

Let $D^{2} \subset \mathbb{R}^{2}$ be the 2-disk. The starting point of the theory of divides ([AC98]) is to describe the 3 -sphere $S^{3}$ in terms of tangent vectors on $D^{2}$ as follows.

$$
\left\{\left.(\boldsymbol{x}, \boldsymbol{y})\left|\boldsymbol{x} \in D^{2}, \boldsymbol{y} \in T_{\boldsymbol{x}} \mathbb{R}^{2},\right| \boldsymbol{x}\right|^{2}+|\boldsymbol{y}|^{2}=1\right\},
$$

where $\boldsymbol{x}=\left(x_{1}, x_{2}\right), \boldsymbol{y}=\left(y_{1}, y_{2}\right)$, and $|\boldsymbol{x}|=\sqrt{x_{1}^{2}+x_{2}^{2}}$. We will also identify $T \mathbb{R}^{2}$ with $\mathbb{C}^{2}$ by $(\boldsymbol{x}, \boldsymbol{y}) \longmapsto \boldsymbol{x}+\sqrt{-1} \boldsymbol{y}$. Therefore, we sometimes call $\boldsymbol{x}$ (resp. $\boldsymbol{y}$ ) the real part (resp. imaginary part) of $(\boldsymbol{x}, \boldsymbol{y})$.

In this paper, we will use the following piecewise linear (rectangular) model for $S^{3}$. For $\boldsymbol{x}=\left(x_{1}, x_{2}\right)$, let us denote the maximum norm by $\|\boldsymbol{x}\|_{\infty}:=\max \left\{\left|x_{1}\right|,\left|x_{2}\right|\right\}$. Let $R>0$ be a positive real number. The rectangle $\operatorname{Rect}(R, 1)$ is defined as

$$
\begin{aligned}
\operatorname{Rect}(R, 1): & =[-R, R] \times[-1,1] \\
& =\left\{\boldsymbol{x}=\left(x_{1}, x_{2}\right) \in \mathbb{R}^{2} \mid-R \leq x_{1} \leq R,-1 \leq x_{2} \leq 1\right\} .
\end{aligned}
$$

For $\boldsymbol{x} \in \operatorname{Rect}(R, 1)$, let $\delta(\boldsymbol{x})$ be the distance between $\boldsymbol{x}$ and the boundary $\partial \operatorname{Rect}(R, 1)$ with respect to the norm $\|\cdot\|_{\infty}$. In other words, $\delta(\boldsymbol{x})=\min \left\{x_{1}+R, R-x_{1}, x_{2}+1,1-x_{2}\right\}$. We will use the following piecewise linear presentation $\mathrm{S}^{3}(R, 1)$ of the sphere (see Figure 1).

$$
S^{3} \approx \mathrm{S}^{3}(R, 1):=\left\{(\boldsymbol{x}, \boldsymbol{y}) \mid \boldsymbol{x} \in \operatorname{Rect}(R, 1), \boldsymbol{y} \in T_{\boldsymbol{x}} \mathbb{R}^{2},\|\boldsymbol{y}\|_{\infty}=\delta(\boldsymbol{x})\right\}
$$

Next, we define a special family of circles which are defined as images of piecewise linear maps from the boundary of the square $\partial \operatorname{Rect}(1,1)$. Let $\boldsymbol{a}_{1}=\left(a_{1}, b\right), \boldsymbol{a}_{2}=\left(a_{2}, b\right) \in \operatorname{Rect}(R, 1)$ be two points with the same height $b$. For simplicity, we assume $a_{1} \leq a_{2}, 0 \leq\left|a_{1}\right|,\left|a_{2}\right| \ll R$, and $0 \leq b<1$. We decompose the boundary $\partial \operatorname{Rect}(1,1)$ into four edges $R_{1}, R_{2}, R_{3}, R_{4}$ as shown in Figure 2, 


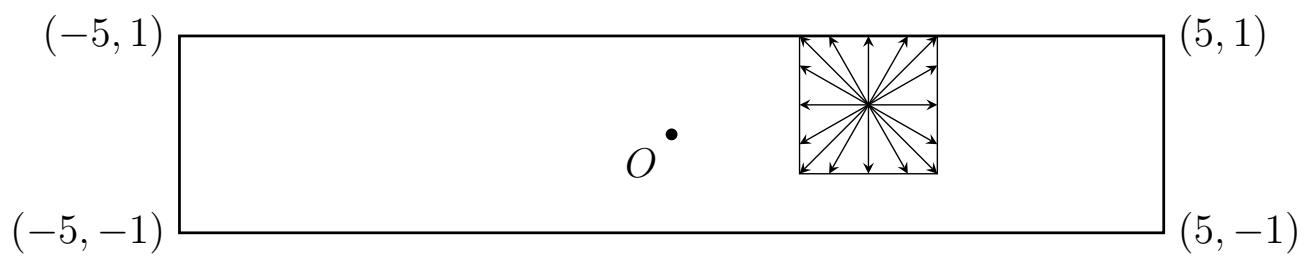

Figure 1: $\operatorname{Rect}(5,1)$ and a circle $\mathbb{S}(\boldsymbol{a}, \boldsymbol{a})$ with $\boldsymbol{a}=(2,0.3)$

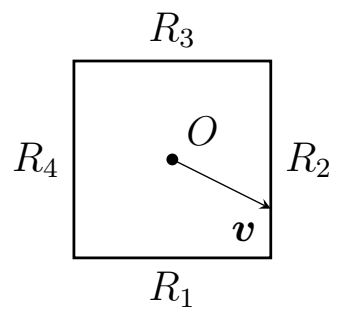

Figure 2: decomposition into $R_{1}, \ldots, R_{4}$

Let $\boldsymbol{v}=\left(v_{1}, v_{2}\right) \in \partial \operatorname{Rect}(1,1)=R_{1} \cup R_{2} \cup R_{3} \cup R_{4}$. Define the map $F: \partial \operatorname{Rect}(1,1) \longrightarrow \mathrm{S}^{3}(R, 1)$ as follows.

$$
F(\boldsymbol{v})= \begin{cases}\left(\left(\frac{a_{2}-a_{1}}{2} v_{1}+\frac{a_{1}+a_{2}}{2}, b\right),(1-b) \boldsymbol{v}\right), & v_{2}=-1\left(R_{1}\right), \\ \left(\left(\frac{a_{1}-a_{2}}{2} v_{2}+\frac{a_{1}+a_{2}}{2}, b\right),(1-b) \boldsymbol{v}\right), & v_{1}=1\left(R_{2}\right), \\ \left(\left(\frac{a_{1}-a_{2}}{2} v_{1}+\frac{a_{1}+a_{2}}{2}, b\right),(1-b) \boldsymbol{v}\right), & v_{2}=1\left(R_{3}\right), \\ \left(\left(\frac{a_{2}-a_{1}}{2} v_{2}+\frac{a_{1}+a_{2}}{2}, b\right),(1-b) \boldsymbol{v}\right), & v_{1}=-1\left(R_{4}\right),\end{cases}
$$

(See also Figure 3). We denote the image of this map by

$$
\mathbb{S}\left(\boldsymbol{a}_{1}, \boldsymbol{a}_{2}\right)=F\left(R_{1}\right) \cup F\left(R_{2}\right) \cup F\left(R_{3}\right) \cup F\left(R_{4}\right) .
$$

Since the argument of the tangent vector is preserved, $\mathbb{S}\left(\boldsymbol{a}_{1}, \boldsymbol{a}_{2}\right)$ is an embedded (piecewise linear) circle. When $\boldsymbol{a}_{1}=\boldsymbol{a}_{2}, \mathbb{S}(\boldsymbol{a}, \boldsymbol{a})$ is a circle (square) as in Figure 1,
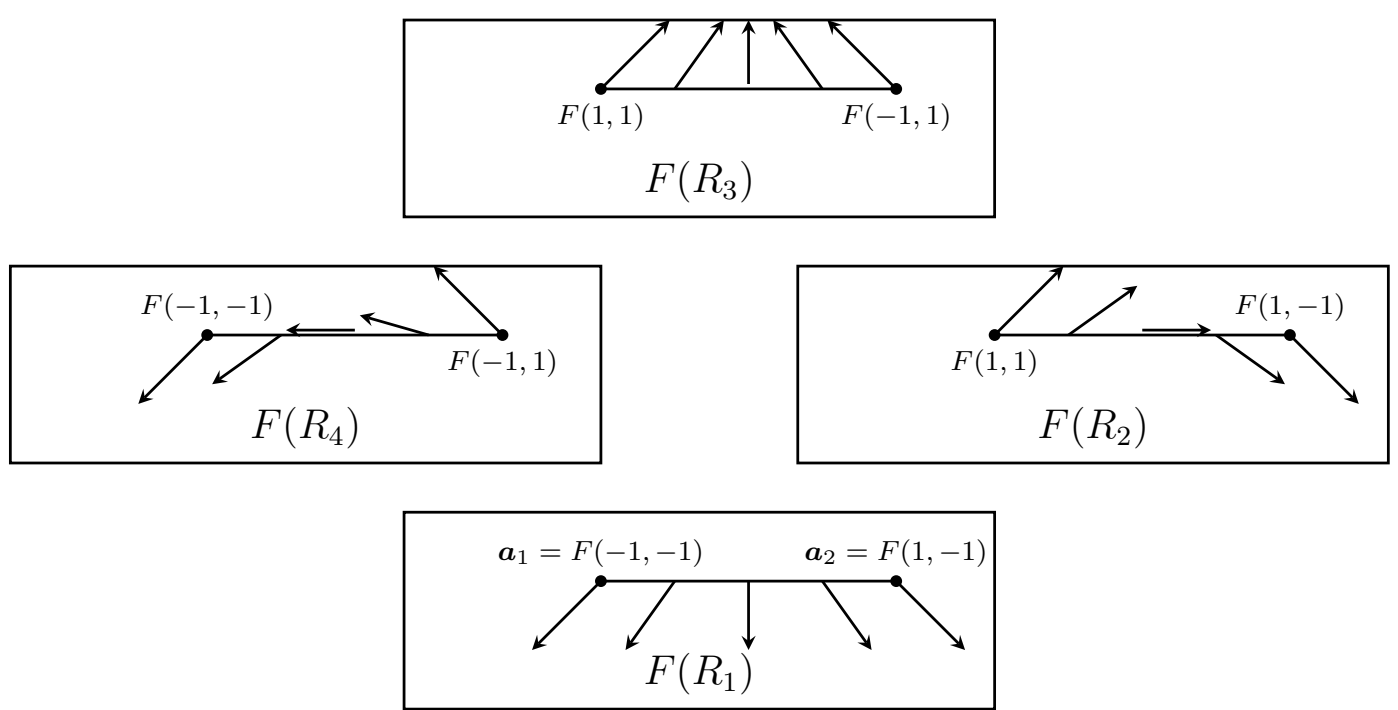

Figure 3: $F\left(R_{1}\right), F\left(R_{2}\right), F\left(R_{3}\right)$, and $F\left(R_{4}\right)$

For simplicity, we draw $\mathbb{S}\left(\boldsymbol{a}_{1}, \boldsymbol{a}_{2}\right)$ as in Figure 4 .

Proposition 2.1. $\mathbb{S}\left(\boldsymbol{a}_{1}, \boldsymbol{a}_{2}\right)$ is isotopic to $\mathbb{S}\left(\boldsymbol{a}_{1}, \boldsymbol{a}_{1}\right)$. Furthermore, $\mathbb{S}\left(\boldsymbol{a}_{1}, \boldsymbol{a}_{2}\right)$ is a trivial knot. 


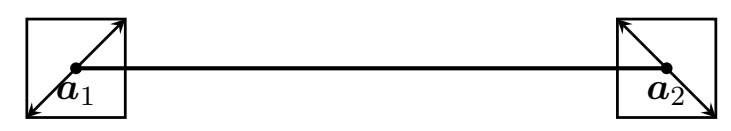

Figure 4: Pictorial notation for $\mathbb{S}\left(\boldsymbol{a}_{1}, \boldsymbol{a}_{2}\right)=F\left(R_{1}\right) \cup F\left(R_{2}\right) \cup F\left(R_{3}\right) \cup F\left(R_{4}\right)$

Proof. It is easily seen that $\mathbb{S}\left(\boldsymbol{a}_{1}, t \boldsymbol{a}_{1}+(1-t) \boldsymbol{a}_{2}\right), 0 \leq t \leq 1$, gives an isotopy and $\mathbb{S}\left(\boldsymbol{a}_{1}, \boldsymbol{a}_{1}\right) \subset$ $\mathrm{S}^{3}(R, 1)$ is trivial.

Remark 2.2. Note that $\mathbb{S}\left(\boldsymbol{a}_{1}, \boldsymbol{a}_{2}\right)$ can be regarded as a piecewise linear knot with four edges. The triviality of $\mathbb{S}\left(\boldsymbol{a}_{1}, \boldsymbol{a}_{2}\right)$ may also be justified by the fact that every knotted piecewise linear knot has at least six edges ([CM98]).

Proposition 2.3. Let $\boldsymbol{a}_{i}=\left(a_{i}, b\right), \boldsymbol{a}_{i}^{\prime}=\left(a_{i}, b^{\prime}\right),(i=1,2)$. Assume $b \neq b^{\prime}$. Then $\mathbb{S}\left(\boldsymbol{a}_{1}, \boldsymbol{a}_{2}\right) \cap$ $\mathbb{S}\left(\boldsymbol{a}_{1}^{\prime}, \boldsymbol{a}_{2}^{\prime}\right)=\emptyset$. Furthermore, $\mathbb{S}\left(\boldsymbol{a}_{1}, \boldsymbol{a}_{2}\right)$ and $\mathbb{S}\left(\boldsymbol{a}_{1}^{\prime}, \boldsymbol{a}_{2}^{\prime}\right)$ are not linked. In particular, the linking number is $\operatorname{Lk}\left(\mathbb{S}\left(\boldsymbol{a}_{1}, \boldsymbol{a}_{2}\right), \mathbb{S}\left(\boldsymbol{a}_{1}^{\prime}, \boldsymbol{a}_{2}^{\prime}\right)\right)=0$.

Proof. Since they have no common real parts, $\mathbb{S}\left(\boldsymbol{a}_{1}, \boldsymbol{a}_{2}\right) \cap \mathbb{S}\left(\boldsymbol{a}_{1}^{\prime}, \boldsymbol{a}_{2}^{\prime}\right)=\emptyset$ is obvious. We may assume $b>b^{\prime}$. Let $L:=\left\{x_{2}=\frac{b+b^{\prime}}{2}\right\}$ be the horizontal line separating two segments $\left[\boldsymbol{a}_{1}, \boldsymbol{a}_{2}\right]$ and $\left[\boldsymbol{a}_{1}^{\prime}, \boldsymbol{a}_{2}^{\prime}\right]$. Then

$$
\left\{(\boldsymbol{x}, \boldsymbol{y}) \mid \boldsymbol{x} \in L, \boldsymbol{y} \in T_{\boldsymbol{x}} \mathbb{R}^{2},\|\boldsymbol{y}\|_{\infty}=\delta(\boldsymbol{x})\right\}
$$

defines an $S^{2}$ in $\mathbb{S}^{3}(R, 1)$ which separates $\mathbb{S}\left(\boldsymbol{a}_{1}, \boldsymbol{a}_{2}\right)$ and $\mathbb{S}\left(\boldsymbol{a}_{1}^{\prime}, \boldsymbol{a}_{2}^{\prime}\right)$ (Figure 5$)$. Therefore, they are not linked.

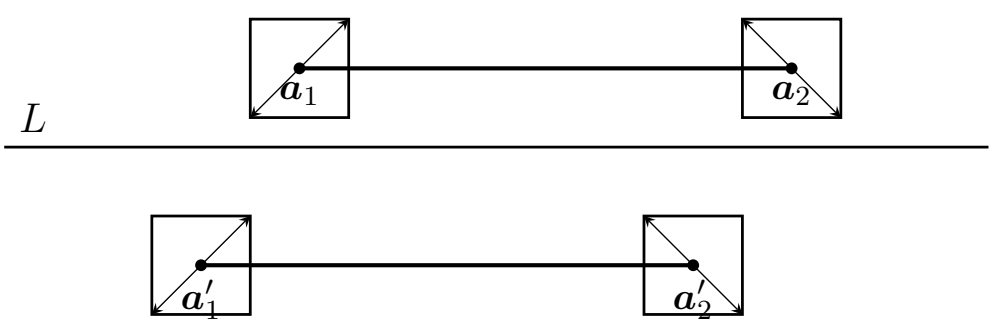

Figure 5: Separation of $\mathbb{S}\left(\boldsymbol{a}_{1}, \boldsymbol{a}_{\mathbf{2}}\right)$ and $\mathbb{S}\left(\boldsymbol{a}_{1}^{\prime}, \boldsymbol{a}_{\mathbf{2}}^{\prime}\right)$

\section{Divides with cusps}

In this section, we introduce the notion of the divide with cusps and associated links.

Let $X$ be a compact 1-dimensional manifold. Then, $X$ is expressed as a disjoint union of finitely many intervals and circles $\bigsqcup_{j} I_{j} \sqcup \bigsqcup_{k} S_{k}$, where $I_{j}$ and $S_{k}$ are intervals and circles, respectively. We have $\partial X=\bigsqcup_{j} \partial I_{j}$.

Definition 3.1. A divide with cusps $C$ is the image of a continuous map $\alpha:(X, \partial X) \longrightarrow$ $\left(D^{2}, \partial D^{2}\right)$ satisfying the following conditions.

(i) $\alpha^{-1}(\partial D)=\partial X,\left.\alpha\right|_{\partial X}$ is injective, and $C$ is transversal to $\partial D$.

(ii) There are finitely many points $p_{1}, \ldots, p_{s}$ in the interior of $X$ such that

- $\alpha$ is an immersion on $X \backslash\left\{p_{1}, \ldots, p_{s}\right\}$ and $\alpha\left(p_{i}\right)$ is a cusp of $C$.

$-\alpha^{-1}\left(\alpha\left(p_{i}\right)\right)=\left\{p_{i}\right\}$.

(iii) Except for cusps and double points, there are no singularities. 


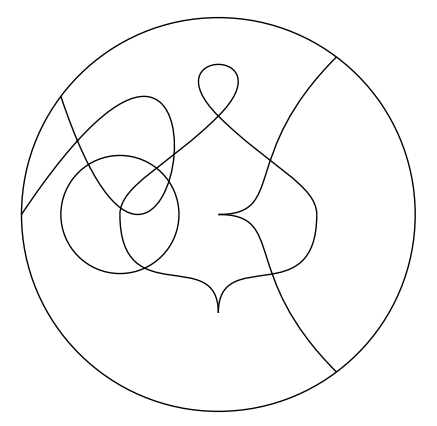

Figure 6: A divide with cusps

(See Figure 6.)

Let $C$ be a divide with cusps. We consider tangent space at $\boldsymbol{x}=\left(x_{1}, x_{2}\right) \in C$. If $\boldsymbol{x}$ is neither double points nor cusps, then $T_{x} C$ is as usual. Note that there is a natural tangent space $T_{x} C$ at a cusp (Figure 7). At a double point, $T_{x} C$ is a union of two tangent spaces.

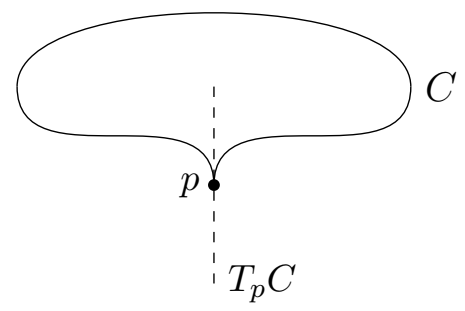

Figure 7: Tangent space $T_{p} C$ at $\operatorname{cusp} p \in C$

To a divide with cusps $C$, the link $L(C)$ is defined as

$$
L(C)=\left\{(\boldsymbol{x}, \boldsymbol{y}),\left.\left|\boldsymbol{x} \in C, \boldsymbol{y} \in T_{\boldsymbol{x}}(C),\right| \boldsymbol{x}\right|^{2}+|\boldsymbol{y}|^{2}=1\right\} \subset S^{3} .
$$

By definition, when $\boldsymbol{x} \in C$ is in the interior of $D^{2}$, there are exactly two tangent vectors $\pm \boldsymbol{y} \in T_{\boldsymbol{x}} C$ which is contained in $L(C)$. The vector $\boldsymbol{y}$ is determined by the argument. Hence (over the interior) the link $L(C)$ can be drawn in $C \times S^{1}$. Note that two kinds of cusps are corresponding to positive and negative half twists (Figure 8 ).

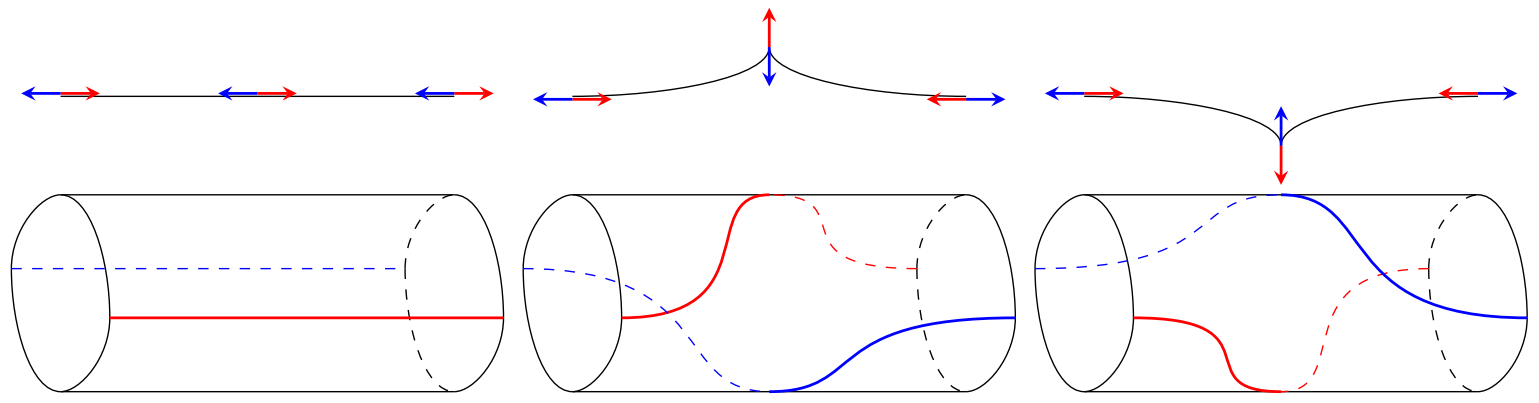

Figure 8: Smooth line, cusps, and corresponding links

Remark 3.2. The moves of divides with cusps in Figure 9 do not change the isotopy type of the corresponding links. The proof is similar to that of [CP00, Lemma 1.3]. 

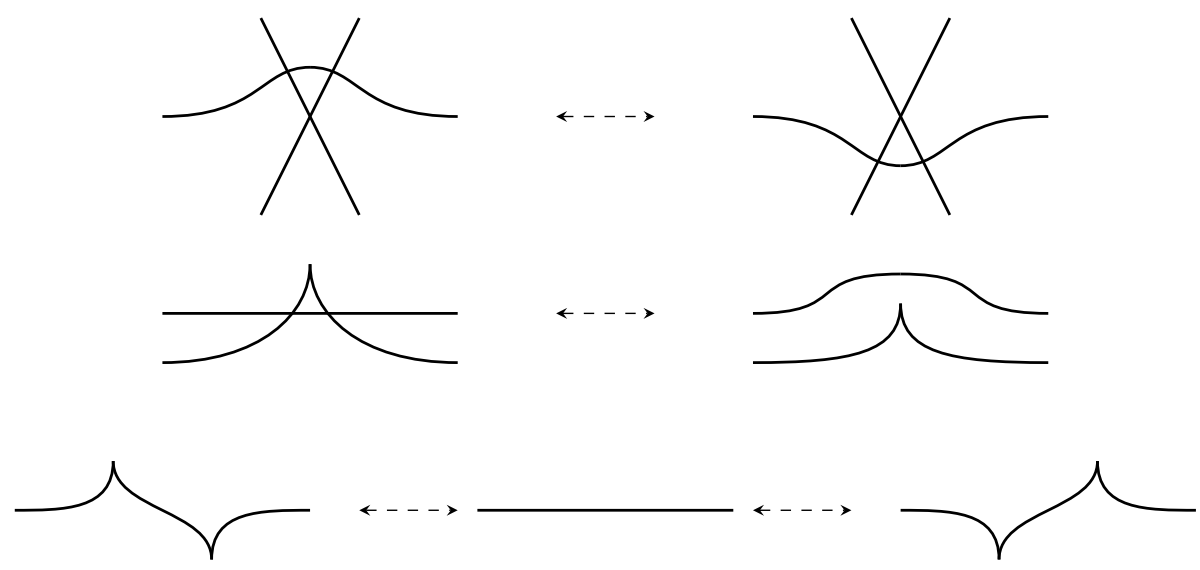

Figure 9: Moves of divides with cusps.

Example 3.3. Let $C_{1}, C_{2}$, and $C_{3}$ be, respectively, a smooth circle, a circle with an outward cusp, and a circle with an inward cusp. Then $L\left(C_{1}\right), L\left(C_{2}\right)$, and $L\left(C_{3}\right)$ are, respectively, the Hopf link, the trivial knot, and the trefoil. (Figure 10, )
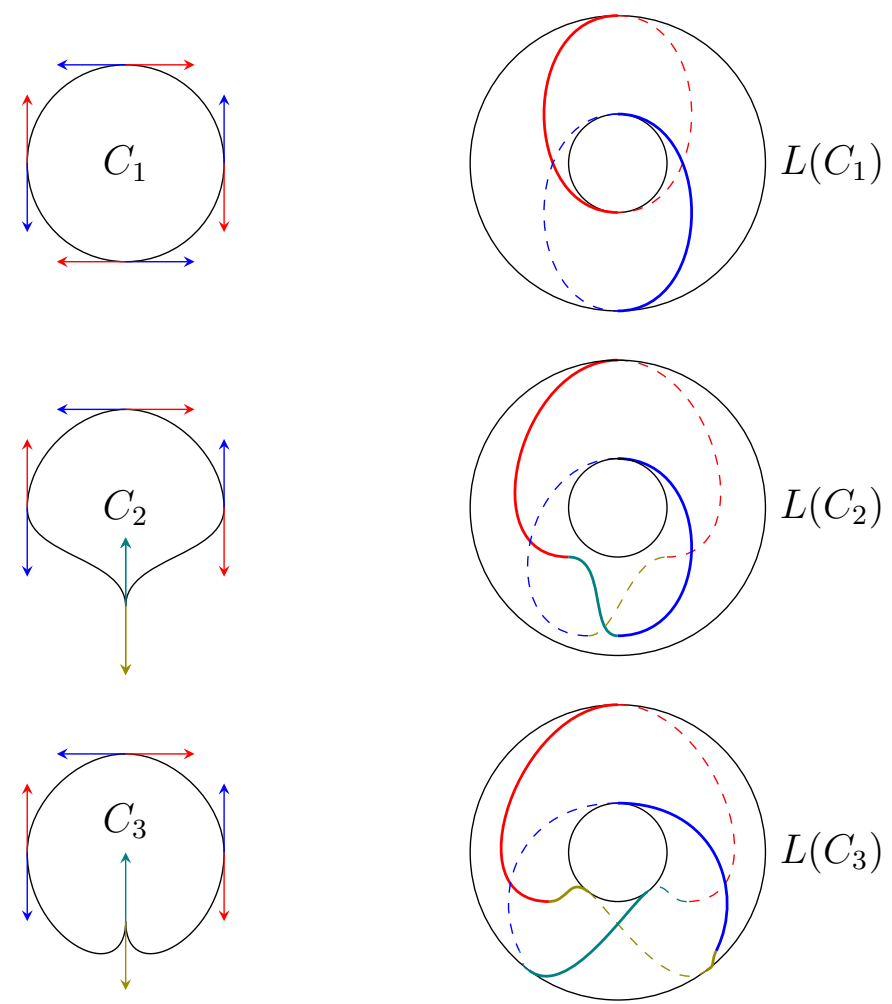

Figure 10: Examples of $L(C)$ (drawn on the torus)

\section{Handle decomposition of $M(\mathcal{A})$}

\subsection{Setting}

A real line arrangement $\mathcal{A}=\left\{H_{1}, \ldots, H_{n}\right\}$ is a finite set of affine lines in the affine plane $\mathbb{R}^{2}$. Each line is defined by some affine linear form

$$
\alpha_{H}\left(x_{1}, x_{2}\right)=a x_{1}+b x_{2}+c=0,
$$

with $a, b, c \in \mathbb{R}$ and $(a, b) \neq(0,0)$. A connected component of $\mathbb{R}^{2} \backslash \bigcup_{H \in \mathcal{A}} H$ is called a chamber. The set of all chambers is denoted by $\operatorname{ch}(\mathcal{A})$. The affine linear equation (2) defines a complex 
line $\left\{\left(z_{1}, z_{2}\right) \in \mathbb{C}^{2} \mid a z_{1}+b z_{2}+c=0\right\}$ in $\mathbb{C}^{2}$. We denote the set of complexified lines by $\mathcal{A}_{\mathbb{C}}=\left\{H_{\mathbb{C}}=H \otimes \mathbb{C} \mid H \in \mathcal{A}\right\}$. The object of our interest is the complexified complement $M(\mathcal{A})=\mathbb{C}^{2} \backslash \bigcup_{H \in \mathcal{A}} H_{\mathbb{C}}$

For a point $p \in \mathbb{R}^{2}$, let $\mathcal{A}_{p}:=\{H \in \mathcal{A} \mid H \ni p\}$. By the identification $T \mathbb{R}^{2} \simeq \mathbb{C}^{2},(\boldsymbol{x}, \boldsymbol{y} \in$ $\left.T_{\boldsymbol{x}} \mathbb{R}^{2}\right) \longmapsto \boldsymbol{x}+\sqrt{-1} \cdot \boldsymbol{y}$, the complexified complement $M(\mathcal{A})$ is the set of all tangent vectors in $\mathbb{R}^{2}$ which are tangent to none of $H \in \mathcal{A}$, namely,

$$
M(\mathcal{A})=\left\{(\boldsymbol{x}, \boldsymbol{y}) \mid \boldsymbol{x} \in \mathbb{R}^{2}, \boldsymbol{y} \in T_{\boldsymbol{x}} \mathbb{R}^{2}, \text { if } H \in \mathcal{A}_{\boldsymbol{x}}, \text { then } \boldsymbol{y} \notin T_{\boldsymbol{x}} H\right\} .
$$

Based on this description, we have the following.

Proposition 4.1. (1) Let $(\boldsymbol{x}, \boldsymbol{y}) \in M(\mathcal{A})$. Then for any $t \in \mathbb{R},(\boldsymbol{x}+t \boldsymbol{y}, \boldsymbol{y}) \in M(\mathcal{A})$.

(2) Suppose $\boldsymbol{y}$ is not parallel to any of $H \in \mathcal{A}$. Then for any $\boldsymbol{x} \in \mathbb{R}^{2},(\boldsymbol{x}, \boldsymbol{y}) \in M(\mathcal{A})$.

Proof. (1) Assume $(\boldsymbol{x}+t \boldsymbol{y}, \boldsymbol{y}) \in H_{\mathbb{C}}$ for some $H \in \mathcal{A}$. Then $\boldsymbol{x}+t \boldsymbol{y} \in H$ and $\boldsymbol{y} \in T_{\boldsymbol{x}+t \boldsymbol{y}} H$ hold. Then $\boldsymbol{y}$ is parallel to $H$, and $\boldsymbol{x} \in H$. Then $(\boldsymbol{x}, \boldsymbol{y}) \in H$ which contradicts $(\boldsymbol{x}, \boldsymbol{y}) \in M(\mathcal{A})$.

(2) is obvious from the description of (3).

Now let us fix a generic line $F$ such that $F$ does not separate intersections of $\mathcal{A}$. We can choose coordinates $x_{1}, x_{2}$ so that $F$ is given by $x_{2}=0$ and all intersections of $\mathcal{A}$ are contained in the upper half-plane $\left\{\left(x_{1}, x_{2}\right) \in \mathbb{R}^{2} \mid x_{2}>0\right\}$. Let

$$
\operatorname{ch}_{F}(\mathcal{A}):=\{C \in \operatorname{ch}(\mathcal{A}) \mid C \cap F=\emptyset\} .
$$

We set $H_{i} \cap F$ has coordinates $\left(a_{i}, 0\right)$. By changing the numbering of lines and signs of the defining equation $\alpha_{i}$ of $H_{i} \in \mathcal{A}$ we may assume that

- $a_{1}<a_{2}<\cdots<a_{n}$, and

- the half-plane $H_{i}^{-}=\left\{\alpha_{i}<0\right\}$ contains the negative direction (leftward) of $F$ (equivalently, $H_{i}^{+}=\left\{\alpha_{i}>0\right\}$ contains the positive direction).

Let us recall the construction in [Y07] briefly. There exists a (after suitable compactification near the boundary) Morse function $\varphi: M(\mathcal{A}) \longrightarrow \mathbb{R}_{\geq 0}$ such that

- $\varphi^{-1}(0)=M(\mathcal{A}) \cap F_{\mathbb{C}}$,

- for each $C \in \operatorname{ch}_{F}(\mathcal{A})$ there is a unique critical point $p_{C} \in C$,

- the chamber $C$ is the stable manifold of $p_{C}$,

- there are no other critical points.

Based on these facts, it was proved that $M(\mathcal{A}) \backslash \bigsqcup_{C \in \operatorname{ch}_{F}(\mathcal{A})} C$ is homeomorphic to an open neighborhood of $F_{\mathbb{C}} \cap M(\mathcal{A})$. The homeomorphism/retraction was originally constructed by using the gradient flow ([Y07, Corollary 5.1.5]). Below we construct a piecewise linear retraction.

After appropriate changes of coordinates, we may further assume the following. (See Figure 11.)

- There exists a positive real number $R_{0}>0$ such that all the intersection points of $\mathcal{A}$ are lying in $\left\{\left(x_{1}, x_{2}\right) \mid 1<x_{2}<R_{0}\right\}$.

- Denote by $\theta_{i}$ the angle between the $x_{1}$-axis (from the positive side) and $H_{i}$. Then $\frac{\pi}{4} \leq \theta_{1} \leq$ $\theta_{2} \leq \cdots \leq \theta_{n} \leq \frac{3 \pi}{4}$.

- Take a sufficiently large real number $R>0\left(R \gg R_{0}\right)$ so that all intersections $H_{i} \cap\left\{x_{2}=\right.$ $\left.R_{0}\right\}, i=1, \ldots, n$, are contained in $\left\{-R+R_{0}<x_{1}<R-R_{0}\right\}$. 


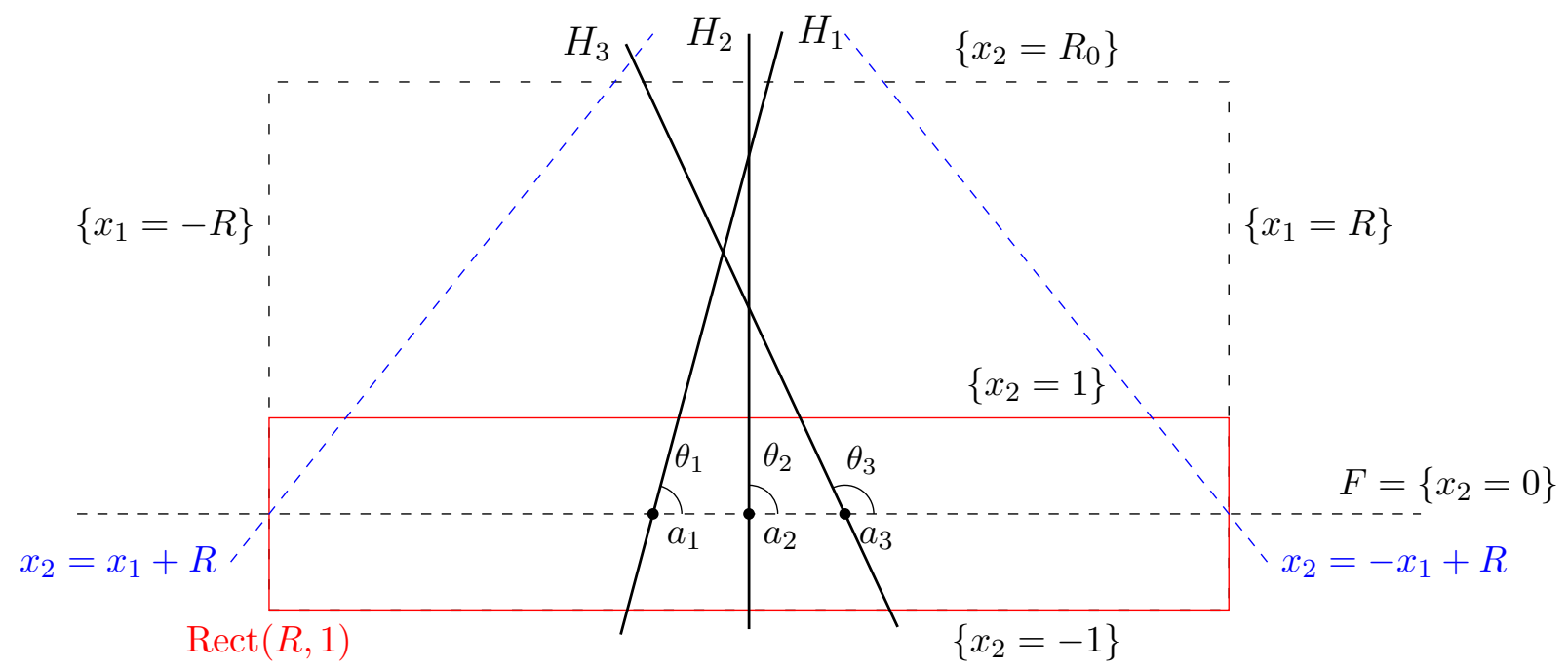

Figure 11: Setting

Let $\widetilde{H}_{\mathbb{C}}$ be a small tubular neighborhood of $H_{\mathbb{C}}$. Define $M_{1}$ as

$$
M_{1}=\left(\mathbb{C}^{2} \backslash \bigcup_{H \in \mathcal{A}} \widetilde{H}_{\mathbb{C}}\right) \cap\left\{(\boldsymbol{x}, \boldsymbol{y}) \mid \boldsymbol{x} \in[-R, R] \times\left[-1, R_{0}\right],\|\boldsymbol{y}\|_{\infty} \leq R\right\},
$$

which is a compact 4-dimensional manifold with boundary whose interior is homeomorphic to $M(\mathcal{A})$ (see [Dur83]).

Next, we remove tubular neighborhoods of each chamber $C$. Let $\widetilde{C}$ be the tubular neighborhood of $C$ in $M(\mathcal{A})$. Define $M_{2}$ as

$$
M_{2}=\left(M_{1} \backslash \bigcup_{C \in \operatorname{ch}(\mathcal{A})} \widetilde{C}\right) \cup\left\{(\boldsymbol{x}, \boldsymbol{y}) \mid \boldsymbol{x} \in \operatorname{Rect}(R, 1),\|\boldsymbol{y}\|_{\infty} \leq R\right\} \backslash \bigcup_{H \in \mathcal{A}} \widetilde{H}_{\mathbb{C}}
$$

Note that if $C \cap F \neq \emptyset$, then the addition of $\widetilde{C}$ does not change the topology. On the other hand, $\widetilde{C}$ for $C \in \operatorname{ch}_{F}(\mathcal{A})$ corresponds to a 2-handle. Hence $M(\mathcal{A})$ is obtained from $M_{2}$ by attaching 2-handles $\widetilde{C}$ for each $C \in \operatorname{ch}_{F}(\mathcal{A})$.

Let $\rho:\left[1, R_{0}\right] \stackrel{\simeq}{\longrightarrow}[0,1]$ be the function defined by $\rho(h)=\frac{h-1}{R_{0}-1}$. Next, we define

$$
M_{3}=\left\{\begin{array}{l|l}
(\boldsymbol{x}, \boldsymbol{y}) \in M_{2} & \begin{array}{l}
\text { If } x_{2} \geq 1, \text { then } \\
x_{2} \leq x_{1}+R, x_{2} \leq-x_{1}+R, \\
\text { and } \rho\left(x_{2}\right) \leq\|\boldsymbol{y}\|_{\infty} \leq 1
\end{array}
\end{array}\right\} .
$$

Note that the inequalities $x_{2} \leq x_{1}+R, x_{2} \leq-x_{1}+R$ mean that the real part of the point is below the blue dashed lines in Figure 11. Since $M_{3}$ is obtained from $M_{2}$ just by deforming near the boundary, $M_{2}$ and $M_{3}$ are homeomorphic. Let us define $M_{4}$ as

$$
M_{4}:=\left\{(\boldsymbol{x}, \boldsymbol{y}) \mid \boldsymbol{x} \in \operatorname{Rect}(R, 1), \boldsymbol{y} \in T_{\boldsymbol{x}} \mathbb{R}^{2},\|\boldsymbol{y}\|_{\infty} \leq \delta(\boldsymbol{x})\right\} \backslash \bigcup_{H \in \mathcal{A}} \widetilde{H}_{\mathbb{C}}
$$

(c. f. Figure 12, )

Now we will construct an explicit contraction from $M_{3}$ to $M_{4}$. 

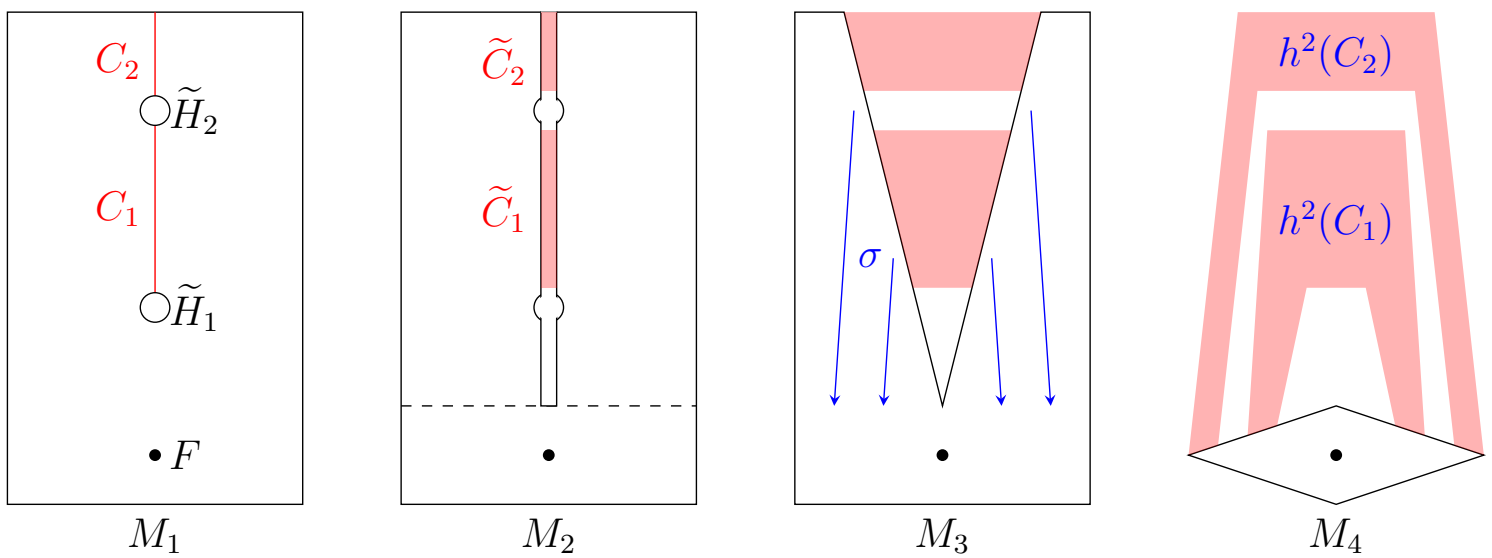

Figure 12: $M_{1}, M_{2}, M_{3}$, and $M_{4}$ (and attached 2-handles).

\subsection{Explicit contraction}

As we noted, $M_{2}, M_{3}$, and $M_{4}$ are homeomorphic. Define the map $\sigma: M_{3} \longrightarrow M_{4}$ by

$$
\sigma(\boldsymbol{x}, \boldsymbol{y}):= \begin{cases}\left(\left(x_{1}-\frac{y_{1}}{y_{2}}\left(x_{2}-1+\|\boldsymbol{y}\|_{\infty}\right), 1-\|\boldsymbol{y}\|_{\infty}\right), \boldsymbol{y}\right), & \text { if } x_{2} \geq 1-\|\boldsymbol{y}\|_{\infty} \text { and }\left|y_{1}\right| \leq\left|y_{2}\right|, \\ \left(\left(x_{1}-\frac{y_{2}}{y_{1}}\left(x_{2}-1+\|\boldsymbol{y}\|_{\infty}\right), 1-\|\boldsymbol{y}\|_{\infty}\right), \boldsymbol{y}\right), & \text { if } x_{2} \geq 1-\|\boldsymbol{y}\|_{\infty} \text { and }\left|y_{1}\right| \geq\left|y_{2}\right|, \\ (\boldsymbol{x}, \boldsymbol{y}) & \text { if } x_{2} \leq 1-\|\boldsymbol{y}\|_{\infty},\end{cases}
$$

where $\boldsymbol{x}=\left(x_{1}, x_{2}\right)$ and $\boldsymbol{y}=\left(y_{1}, y_{2}\right)$ (Figure 13). Note that the first case is for the edges $R_{1}$ and $R_{3}$, the second case is for the edges $R_{2}$ and $R_{4}$, and the third case is for $(\boldsymbol{x}, \boldsymbol{y}) \in M_{4}$.

Let $\sigma_{t}(\boldsymbol{x}, \boldsymbol{y})=(1-t) \cdot(\boldsymbol{x}, \boldsymbol{y})+t \cdot \sigma(\boldsymbol{x}, \boldsymbol{y})$. It is straightforward that if $(\boldsymbol{x}, \boldsymbol{y}) \in M_{3}$, then $\sigma_{t}(\boldsymbol{x}, \boldsymbol{y}) \in M_{3}$ for $0 \leq t \leq 1$. Since $\sigma_{0}$ is the identity on $M_{3}$ and $\sigma_{1}=\sigma, \sigma_{t}(0 \leq t \leq 1)$ gives a contraction of $M_{3}$ to $M_{4}$.

\subsection{Attaching maps of 2-handles}

In this section, we describe the Kirby diagrams (the framed links of attaching circles and the boundaries of carved disks) associated with the handle decompositions obtained in the previous section. Our 0-handle, namely $D^{4}$, is the piecewise linear disk that we introduced in $\$ 2$

$$
\mathrm{D}^{4}(R, 1) \approx\left\{(\boldsymbol{x}, \boldsymbol{y}) \mid \boldsymbol{x} \in \operatorname{Rect}(R, 1), \boldsymbol{y} \in T_{\boldsymbol{x}} \mathbb{R}^{2},\|\boldsymbol{y}\|_{\infty} \leq \delta(\boldsymbol{x})\right\} .
$$

Note that $\partial \mathrm{D}^{4}(R, 1)=\mathrm{S}^{3}(R, 1)$ and $\mathrm{D}^{4}(R, 1) \cap M_{1}=M_{4}$.

Next, we consider 1-handlebody. Note that the intersection $\mathrm{D}^{4}(R, 1) \cap M_{1}$ is equal to $\mathrm{D}^{4}(R, 1) \backslash$ $\bigsqcup_{i=1} \widetilde{H}_{i, \mathbb{C}}$. The intersection $\mathrm{D}^{4}(R, 1) \cap H_{i, \mathbb{C}}$ is homeomorphic to a disk whose boundary $\partial\left(\mathrm{D}^{4}(R, 1) \cap\right.$ $\left.H_{i, \mathbb{C}}\right)=\mathrm{S}^{3}(R, 1) \cap H_{i, \mathbb{C}}$ is a circle. This circle is presented as "dotted circle" in the Kirby diagram ([Ak16, K89, GS99]). We note that $\mathrm{S}^{3}(R, 1) \cap H_{i, \mathbb{C}}$ is the knot corresponding to the divide $\bar{H}_{i}:=H_{i} \cap \operatorname{Rect}(R, 1)$. Thus the Kirby diagram of the 1 -handlebody is the link associated with the divide consisting of $n$ intervals $\left\{\overline{H_{1}}, \ldots, \bar{H}_{n}\right\}$ in $\operatorname{Rect}(R, 1)$ (Figure 14).

From the description in the previous section, to each chamber $C \in \operatorname{ch}_{F}(\mathcal{A})$, the union of the neighborhood of $C$

$$
\left\{(\boldsymbol{x}, \boldsymbol{y}) \mid \boldsymbol{x} \in C,\|\boldsymbol{y}\|_{\infty} \leq \rho\left(x_{2}\right)\right\}
$$

and the trajectories of the contraction

$$
\left\{\sigma_{t}(\boldsymbol{x}, \boldsymbol{y}) \mid \boldsymbol{x} \in C,\|\boldsymbol{y}\|_{\infty}=\rho\left(x_{2}\right), 0 \leq t \leq 1\right\}
$$

gives an explicit 2-handle $h^{2}(C)$ attached to $M_{4}$ (Figure 12). 


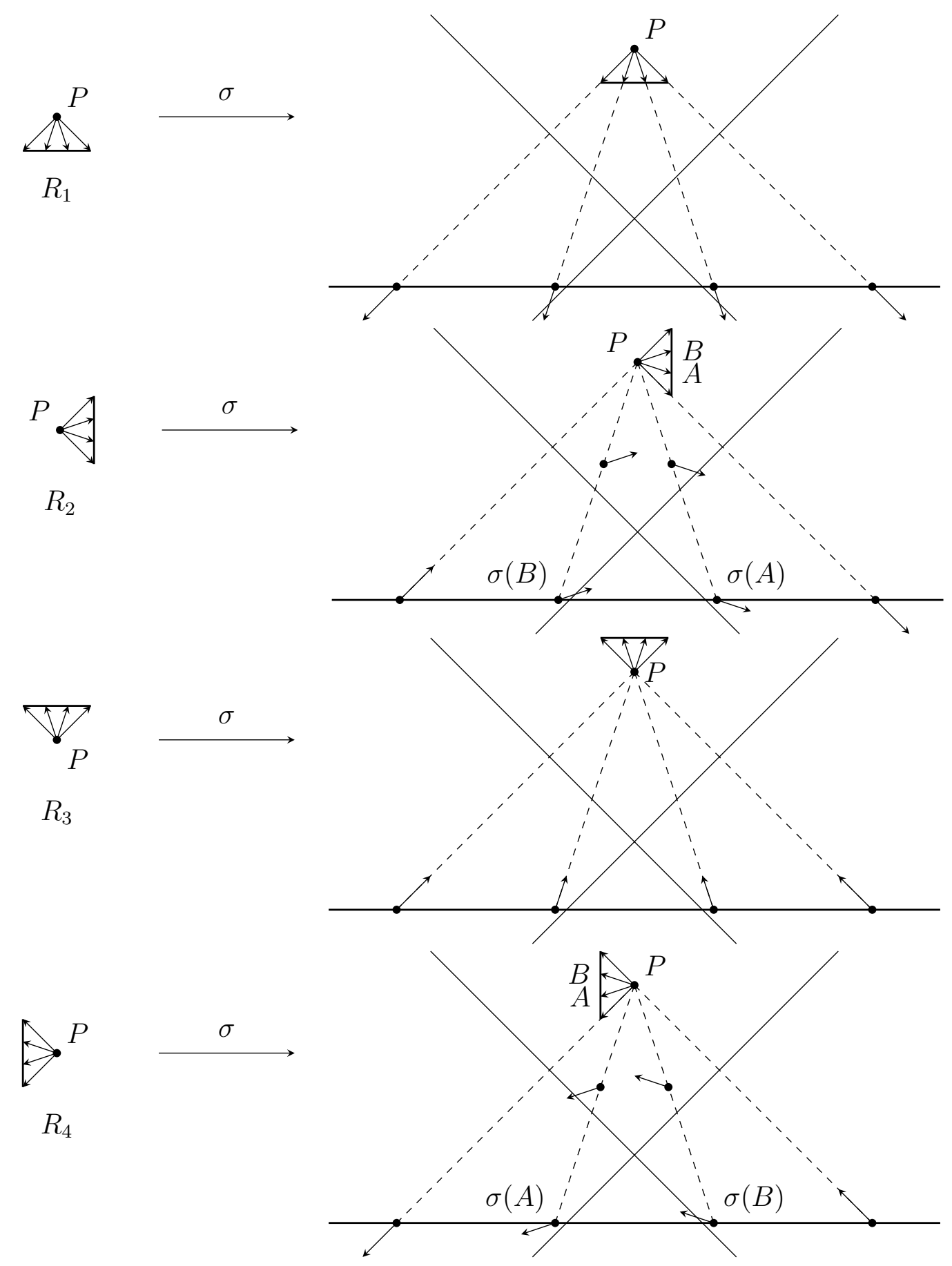

Figure 13: $\sigma: M_{3} \rightarrow M_{4}$

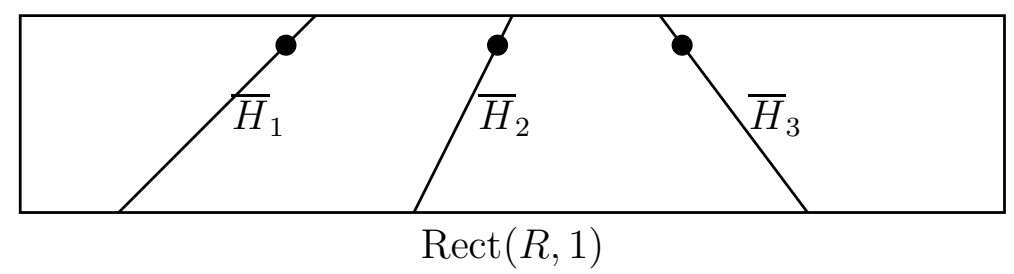

Figure 14: Divide whose link (with dots) represents the 1-handlebody 
Let $P=\left(x_{1}, x_{2}\right) \in C$. The real part of $\sigma\left(x_{1}, x_{2},-\rho\left(x_{2}\right),-\rho\left(x_{2}\right)\right)$ is $\left(x_{1}-x_{2}+1-\rho\left(x_{2}\right), 1-\rho\left(x_{2}\right)\right)$ which we denote by $\boldsymbol{a}_{1}(P)$. Similarly, the real part of $\sigma\left(x_{1}, x_{2}, \rho\left(x_{2}\right),-\rho\left(x_{2}\right)\right)$ is $\left(x_{1}+x_{2}-1+\right.$ $\left.\rho\left(x_{2}\right), 1-\rho\left(x_{2}\right)\right)$ which we denote by $\boldsymbol{a}_{2}(P)$.

Theorem 4.2. The attaching circle of the 2-handle $h^{2}(C)$ for $C \in \operatorname{ch}_{F}(\mathcal{A})$ is equal to $\mathbb{S}\left(\boldsymbol{a}_{1}, \boldsymbol{a}_{2}\right)$ with zero framing. (Figure 15)

Proof. The first assertion is clear from the definition of $\sigma$. The framing is computed as the linking number of $\mathbb{S}\left(\boldsymbol{a}_{1}(P), \boldsymbol{a}_{2}(P)\right)$ and $\mathbb{S}\left(\boldsymbol{a}_{1}\left(P^{\prime}\right), \boldsymbol{a}_{2}\left(P^{\prime}\right)\right)$, where $P^{\prime}=P+(0, \varepsilon)$. By Proposition 2.3, the linking number is zero.

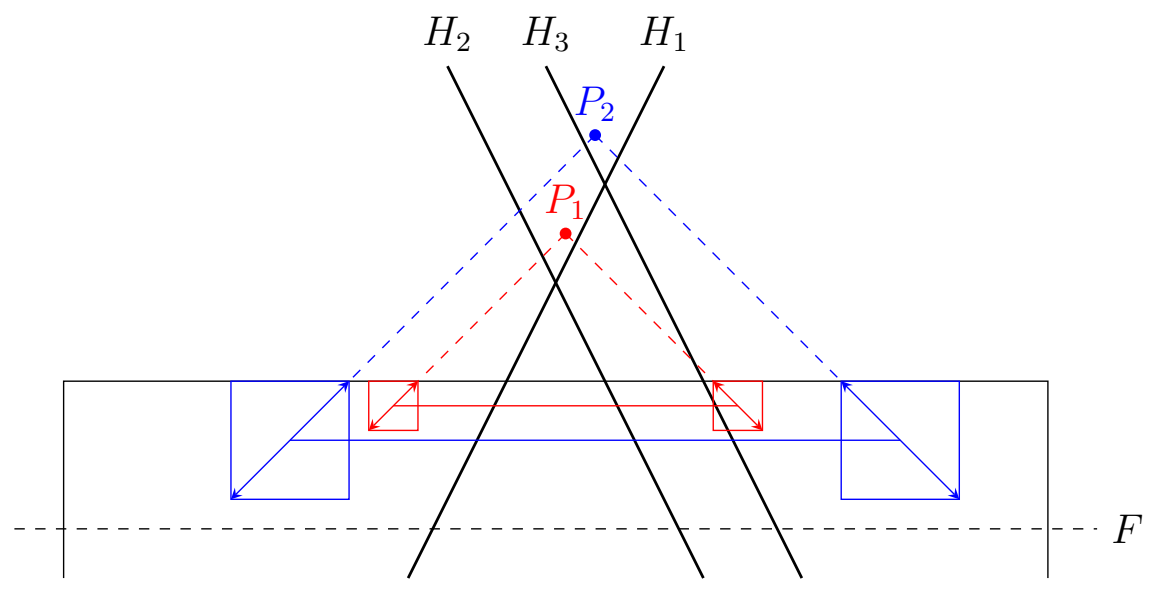

Figure 15: Attaching circles $\mathbb{S}\left(\boldsymbol{a}_{1}\left(P_{i}\right), \boldsymbol{a}_{2}\left(P_{i}\right)\right)$

In the next section, we describe $\mathbb{S}\left(\boldsymbol{a}_{1}(P), \boldsymbol{a}_{2}(P)\right)$ as a link associated with a divide with cusps.

\section{$5 \quad$ Kirby diagrams using divides with cusps}

\subsection{Main result}

Let $b=\left|\operatorname{ch}_{F}(\mathcal{A})\right|$ and $\operatorname{ch}_{F}(\mathcal{A})=\left\{C_{1}, \ldots, C_{b}\right\}$. For each $1 \leq s \leq b$, choose a point $P_{s}=\left(k_{s}, h_{s}\right) \in$ $C_{s}$. We may assume that $1<h_{1}<\cdots<h_{b}<R_{0}$. We also choose $0<h_{s}^{\prime}<1$ and $\varepsilon>0$ (sufficiently small) such that $1>h_{1}^{\prime}>\cdots>h_{b}^{\prime}>0,\left|h_{s}^{\prime}-h_{s+1}^{\prime}\right|>2 \varepsilon$ and $\varepsilon<h_{b}^{\prime}<h_{1}^{\prime}<1-\varepsilon$. (E.g., $h_{s}^{\prime}=1-\rho\left(h_{s}\right)$ )

We define the $\operatorname{sign} \delta_{i}(C)$ of $C \in \operatorname{ch}_{F}(\mathcal{A})$ with respect to $H_{i}$ as follows.

$$
\delta_{i}(C)= \begin{cases}+1, & \text { if } \alpha_{i}(C)>0 \\ -1, & \text { if } \alpha_{i}(C)<0\end{cases}
$$

We note that $\delta_{i}(C)=+1$ (resp. $\delta_{i}(C)=-1$ ) is equivalent to that $C$ is sitting in the right (resp. left) side of $H_{i}$. To each $C=C_{i} \in \operatorname{ch}_{F}(\mathcal{A})$, define a curve $\gamma(C)$ with cusps by joining the following parts (these parts are drawn in the strip $h_{i}^{\prime}-\varepsilon<x_{2}<h_{i}^{\prime}+\varepsilon$ in $\operatorname{Rect}(R, 1)$ ).

(i) In the left side of $\bar{H}_{1}$, if $\delta_{1}(C)=+1$, then draw a leftward cusp, if $\delta_{1}(C)=-1$, then draw a round curve as in Figure 16 .

(ii) According to $\left(\delta_{i}(C), \delta_{i+1}(C)\right)=(+1,-1),(-1,+1)$, or $\delta_{i}=\delta_{i+1}$, we draw a (two) curves between $H_{i}$ and $H_{i+1}$ as in Figure 17.

(iii) In the right side of $\bar{H}_{n}$, if $\delta_{n}(C)=+1$, then draw a round curve, if $\delta_{n}(C)=-1$, then draw a rightward cusp as in Figure 18 . 

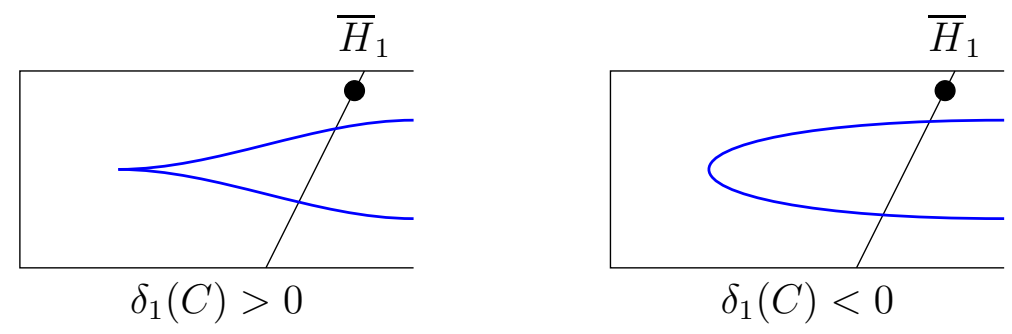

Figure 16: The curve $\gamma(C)$ at the left side of $H_{1}$.
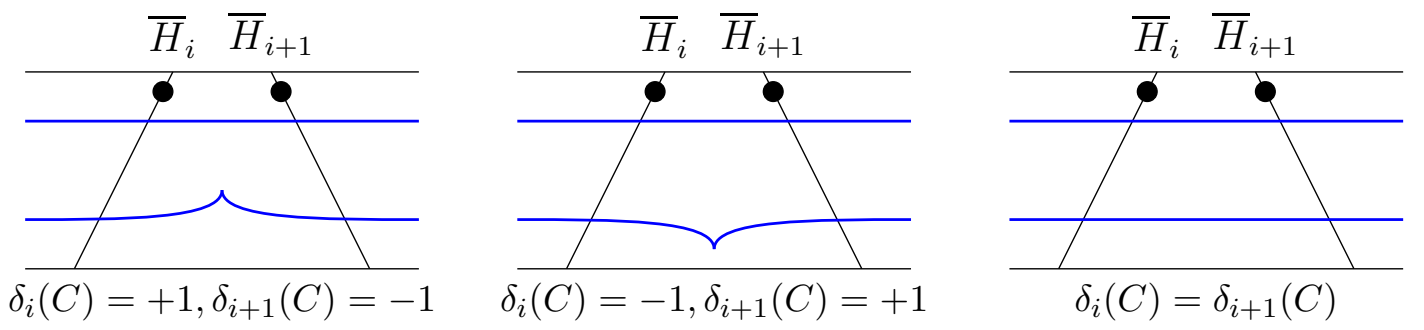

Figure 17: The curve $\gamma(C)$ between $H_{i}$ and $H_{i+1}$.
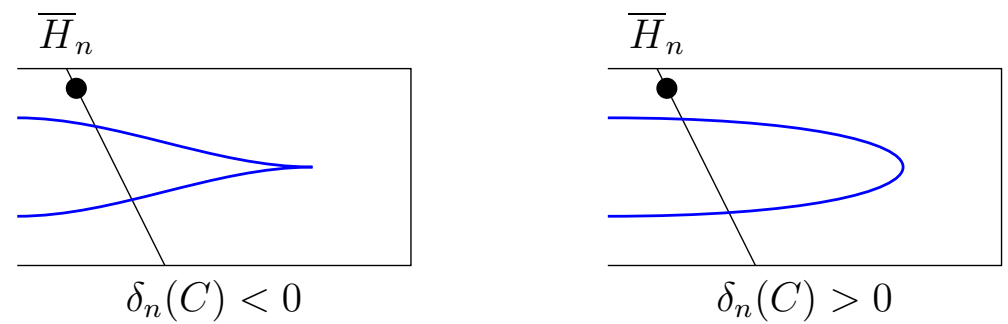

Figure 18: The curve $\gamma(C)$ at the right side of $H_{n}$. 
Theorem 5.1. The link corresponding to $\left\{\gamma\left(C_{1}\right), \ldots, \gamma\left(C_{b}\right)\right\}$ (with zero framing) present the attaching circles of the 2-handles of $M(\mathcal{A})$.

We will prove Theorem 5.1 in $\$ 5.2$,

The curves $\gamma\left(C_{1}\right), \ldots, \gamma\left(C_{b}\right)$ sometimes contain redundant cusps. Therefore, we define reduced curves $\gamma^{\prime}\left(C_{1}\right), \ldots, \gamma^{\prime}\left(C_{b}\right)$ which are obtained from the previous curves by the moves formulated in Remark 3.2.

Definition 5.2. For a chamber $C \in \operatorname{ch}_{F}(\mathcal{A})$, let $m_{1}:=\min \left\{i \mid \delta_{i}(C)=-1\right\}$ and $m_{2}:=$ $\max \left\{i \mid \delta_{i}(C)=+1\right\}$. Let us define the curve $\gamma^{\prime}(C)$ by joining above (i), (ii), (iii) for the lines $H_{m_{1}}, H_{m_{1}+1}, \ldots, H_{m_{2}}$ instead of $H_{1}, \ldots, H_{n}$. We call $\gamma^{\prime}(C)$ the reduced curve of $\gamma(C)$. (Figure 20)

Proposition 5.3. The divides $\left(\gamma^{\prime}(C), \bar{H}_{1}, \ldots, \bar{H}_{n}\right)$ and $\left(\gamma(C), \bar{H}_{1}, \ldots, \bar{H}_{n}\right)$ give isotopic links in $\mathrm{S}^{3}(R, 1)$.

Proof. If $m_{1}=1, m_{2}=n$, then $\gamma^{\prime}(C)=\gamma(C)$. Suppose $m_{1}>1\left(\right.$ or $\left.m_{2}<n\right)$. Then $\delta_{1}(C)=+1$. Hence at the left side of $\bar{H}_{1}, \gamma(C)$ has a leftward cusp. Using the moves in Remark 3.2 (Figure 9), $\gamma(C)$ is deformed to $\gamma^{\prime}(C)$.

Example 5.4. Let us consider 4-lines $\mathcal{A}=\left\{H_{1}, H_{2}, H_{3}, H_{4}\right\}$ as in Figure 19, Let $\operatorname{ch}_{F}(\mathcal{A})=$ $\left\{C_{1}, \ldots, C_{6}\right\}$ and fix $P_{i} \in C_{i}$ as shown in the figure. Then the curves $\gamma_{i}:=\gamma\left(C_{i}\right)(i=1, \ldots, 6)$ are as in Figure 19 (below). The curves $\gamma_{i}, i \geq 3$, are reduced. However, $\gamma_{1}$ and $\gamma_{2}$ have reductions.

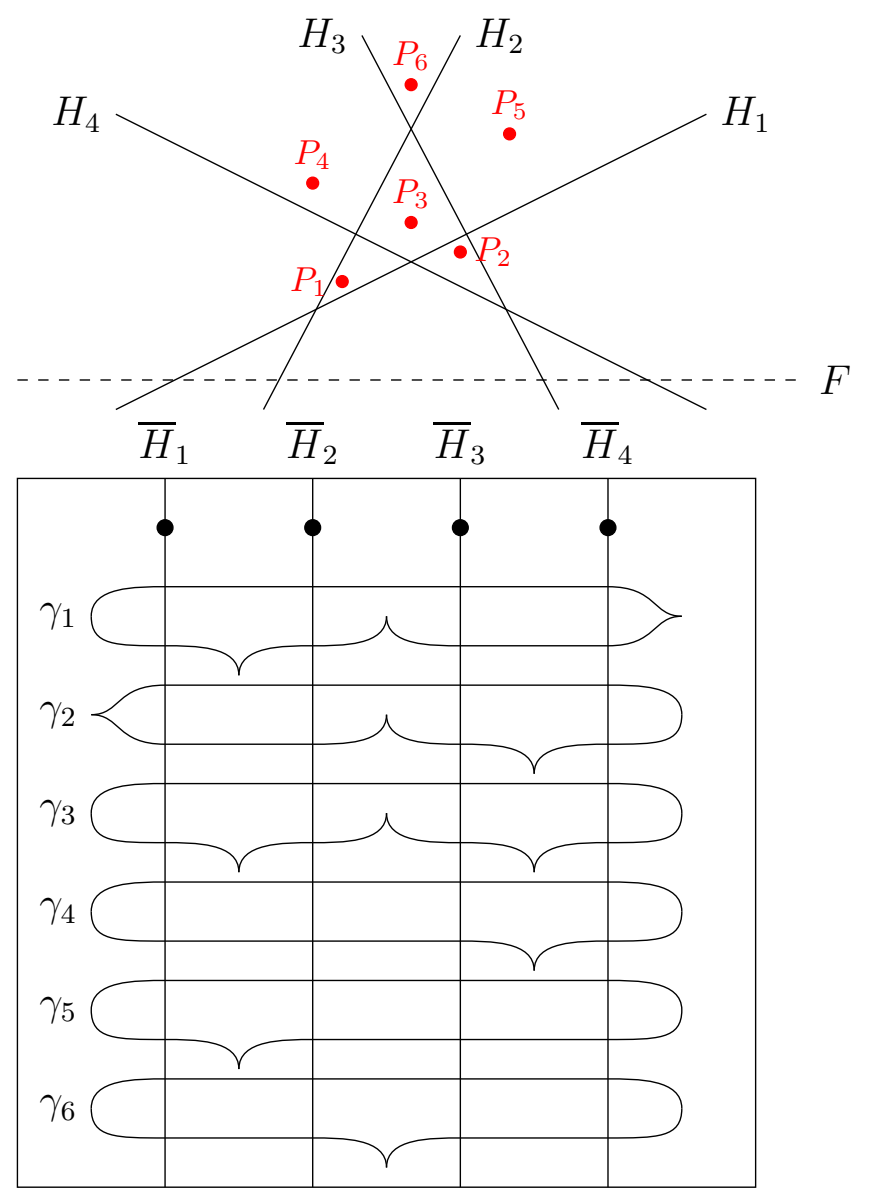

Figure 19: Generic four lines and its divide with cusps

The reduced ones $\gamma_{1}^{\prime}, \gamma_{2}^{\prime}$ are as in Figure 20. 


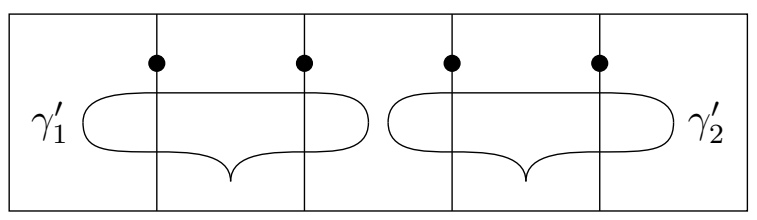

Figure 20: Reduction of $\gamma_{1}$ and $\gamma_{2}$ of Figure 19

\subsection{Proof of Theorem 5.1}

In this section, we prove Theorem [5.1. Let $P \in C$. Here we will show that the link $\mathbb{S}\left(\boldsymbol{a}_{1}(P), \boldsymbol{a}_{2}(P)\right)$ is isotopic to the link determined by the curve constructed as in $\$ 5.1$ between $\boldsymbol{a}_{1}(P)$ and $\boldsymbol{a}_{2}(P)$. The latter can be deformed to $\gamma^{\prime}(C)$ (and to $\gamma(C)$ ) by the moves in Remark 3.2 (Figure 21).
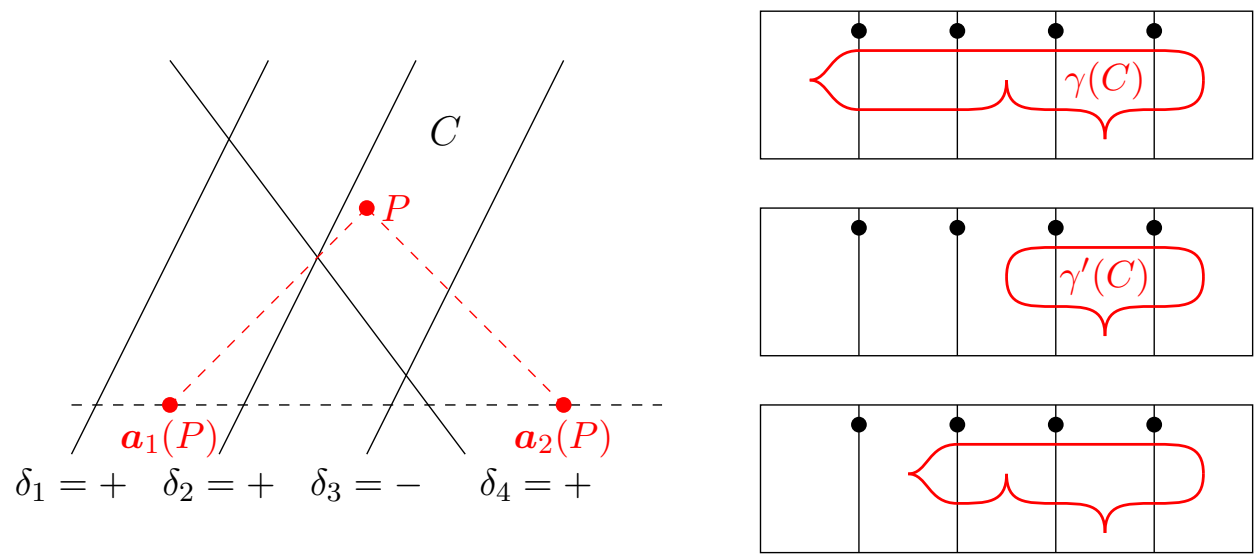

Figure 21: $\gamma(C), \gamma^{\prime}(C)$, and the curve obtained from $\mathbb{S}\left(\boldsymbol{a}_{1}(P), \boldsymbol{a}_{2}(P)\right)$ by the operation below (which is in between $\gamma(C)$ and $\gamma^{\prime}(C)$ ).

First, we consider the image of $R_{2}$ and $R_{4}$ in $\mathbb{S}\left(\boldsymbol{a}_{1}(P), \boldsymbol{a}_{2}(P)\right)$. The argument $\theta$ of tangent vectors satisfies $-\frac{\pi}{4} \leq \theta \leq \frac{\pi}{4}$ (for $R_{2}$ ) or $\frac{3 \pi}{4} \leq \theta \leq \frac{5 \pi}{4}$ (for $R_{4}$ ). Hence by the assumptions on $\mathcal{A}$ (\$4.1), the image of $R_{2}$ is isotopic to the constant vectors with argument $\theta=0$, and that of $R_{4}$ is isotopic to $\theta=\pi$. Therefore, the image of $R_{2} \cup R_{4}$ is isotopic to a part of a simple closed curve corresponding to the tangent vectors of the upper line of $\gamma(C)$.

Next, we consider the marginal case (the case corresponding to Figure 221). Suppose that $\boldsymbol{a}_{1}(P)$ is in between $H_{i-1}$ and $H_{i}$. Let us consider $\sigma\left(R_{4}\right)$ and $\sigma\left(R_{1}\right)$. We shift $\sigma\left(R_{4}\right)$ upward by $\varepsilon$, and $\sigma\left(R_{1}\right)$ downward by $\varepsilon$. By connecting two left most points by a segment with tangent vectors of argument $\frac{5 \pi}{4}$, we obtain a piecewise linear curve $c:[0,1] \longrightarrow \operatorname{Rect}(R, 1) \cap H_{1}^{-}$(red segments in Figure 16) and tangent vectors $\boldsymbol{v}(t) \in T_{\boldsymbol{c}(t)} \mathbb{R}^{2}$. Note that $\{(\boldsymbol{c}(t), \boldsymbol{v}(t)) \mid 0 \leq t \leq 1\}$ forms the $\left(\varepsilon\right.$-shifted) image $\sigma\left(R_{4}\right) \cup \sigma\left(R_{1}\right)$. Now suppose that $\delta_{i}(C)=-1$. Then $\boldsymbol{v}(0)$ is directing the negative half space $H_{i}^{-}$and $\boldsymbol{v}(1)$ is directing the positive half space $H_{i}^{+}$.

There exist a curve $\widetilde{\boldsymbol{c}}(t)$ (the blue curve in Figure 22) and tangent vector $\widetilde{\boldsymbol{v}}(t) \in T_{\widetilde{\boldsymbol{c}}(t)} \mathbb{R}^{2}$ such that

- The curve $\widetilde{C}=\{\widetilde{\boldsymbol{c}}(t) \mid t \in[0,1]\}$ is smooth.

- $\widetilde{\boldsymbol{c}}(0)=\boldsymbol{c}(0), \widetilde{\boldsymbol{c}}(1)=\boldsymbol{c}(1)$.

- $\widetilde{\boldsymbol{v}}(t) \in T_{\widetilde{\boldsymbol{c}}(t)} \widetilde{C}, \widetilde{\boldsymbol{v}}(t) \neq 0$.

- Let $\widetilde{\boldsymbol{v}}(t)=\left(\widetilde{v}_{1}(t), \widetilde{v}_{2}(t)\right)$. Then $\widetilde{v}_{2}(0)=\widetilde{v}_{2}(1)=0, v_{1}(0)<0, v_{1}(1)>0$, and $\widetilde{v}_{2}(t)>0$ for $0<t<1$.

Then we have 
- both $\boldsymbol{v}(0)$ and $\widetilde{\boldsymbol{v}}(0)$ are directing negative side of $H_{i}$,

- both $\boldsymbol{v}(1)$ and $\widetilde{\boldsymbol{v}}(1)$ are directing the positive side of $H_{i}$, and

- for $0<t<1, v_{2}(t)<0$ and $\widetilde{v}_{2}(t)<0$ hold.

Therefore, $s \cdot \widetilde{\boldsymbol{v}}(t)+(1-s) \cdot \boldsymbol{v}(t)$ is nonzero for all $0 \leq s \leq 1$, and $s \cdot(\widetilde{\boldsymbol{c}}(t), \widetilde{\boldsymbol{v}}(t))+(1-s) \cdot(\boldsymbol{c}(t), \boldsymbol{v}(t))$ $(0 \leq s \leq 1)$ gives an isotopy between $(\widetilde{\boldsymbol{c}}(t), \widetilde{\boldsymbol{v}}(t))$ and $(\boldsymbol{c}(t), \boldsymbol{v}(t))$. The other case $\left(\delta_{i}(C)=+1\right)$ is similar.

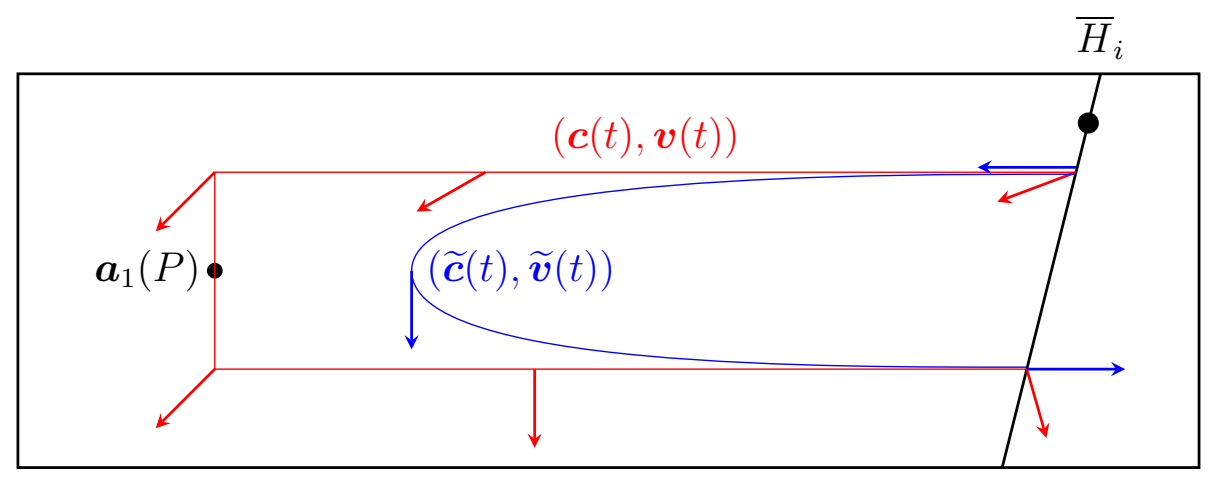

Figure 22: Isotopy between $(\widetilde{\boldsymbol{c}}(t), \widetilde{\boldsymbol{v}}(t))$ and $(\boldsymbol{c}(t), \boldsymbol{v}(t))$

Next, we consider the case $\delta_{i}(C)=+1, \delta_{i+1}(C)=-1$. The intersection $\left[\boldsymbol{a}_{1}(P), \boldsymbol{a}_{2}(P)\right] \cap\left\{\alpha_{i} \geq\right.$ $\left.0, \alpha_{i+1} \leq 0\right\}$ is an interval. Fix a linear parametrization $c:[0,1] \stackrel{\simeq}{\longrightarrow}\left[\boldsymbol{a}_{1}(P), \boldsymbol{a}_{2}(P)\right] \cap\left\{\alpha_{i} \geq\right.$ $\left.0, \alpha_{i+1} \leq 0\right\}$. Then the image of $\sigma\left(R_{3}\right)$ is expressed as $\{(\boldsymbol{c}(t), \boldsymbol{v}(t)) \mid t \in[0,1]\}$, where $\boldsymbol{v}(t) \in$ $T_{\boldsymbol{c}(t)} \mathbb{R}^{2}$ which points to the fixed point $P \in C$ (i.e., $\boldsymbol{v}(t)$ is a positive scalar multiple of the vector $\overrightarrow{\boldsymbol{c}(t) \cdot P})$. In particular, since $\delta_{i}(C)=+1, \boldsymbol{v}(0) \in T_{c(0)} \mathbb{R}^{2}$ is directing to the positive side of $H_{i}$. Similarly, $\boldsymbol{v}(1) \in T_{c(1)} \mathbb{R}^{2}$ is directing to the negative side of $H_{i+1}$. Let $\boldsymbol{v}(t)=\left(v_{1}(t), v_{2}(t)\right)$. Then we also have $v_{2}(t)>0$ for all $t \in[0,1]$. There exists a curve $\widetilde{\boldsymbol{c}}(t)$ and tangent vector $\widetilde{\boldsymbol{v}}(t) \in T_{\boldsymbol{c}(t)} \mathbb{R}^{2}$ such that (Figure 23)

- The curve $\widetilde{C}=\{\widetilde{\boldsymbol{c}}(t) \mid t \in[0,1]\}$ has a unique upward cusp.

- $\widetilde{\boldsymbol{c}}(0)=\boldsymbol{c}(0), \widetilde{\boldsymbol{c}}(1)=\boldsymbol{c}(1)$.

- $\widetilde{\boldsymbol{v}}(t) \in T_{\widetilde{\boldsymbol{c}}(t)} \widetilde{C}$.

- Let $\widetilde{\boldsymbol{v}}(t)=\left(\widetilde{v}_{1}(t), \widetilde{v}_{2}(t)\right)$. Then $\widetilde{v}_{2}(0)=\widetilde{v}_{2}(1)=0$, and $\widetilde{v}_{2}(t)>0$ for $0<t<1$.

Then we have

- both $\boldsymbol{v}(0)$ and $\widetilde{\boldsymbol{v}}(0)$ are directing the positive side of $H_{i}$,

- both $\boldsymbol{v}(1)$ and $\widetilde{\boldsymbol{v}}(1)$ are directing negative side of $H_{i+1}$, and

- for $0<t<1, v_{2}(t)>0$ and $\widetilde{v}_{2}(t)>0$ hold.

Therefore, $s \cdot \widetilde{\boldsymbol{v}}(t)+(1-s) \cdot \boldsymbol{v}(t)$ is nonzero for all $0 \leq s \leq 1$, and $s \cdot(\widetilde{\boldsymbol{c}}(t), \widetilde{\boldsymbol{v}}(t))+(1-s) \cdot(\boldsymbol{c}(t), \boldsymbol{v}(t))$ $(0 \leq s \leq 1)$ gives an isotopy between $(\widetilde{\boldsymbol{c}}(t), \widetilde{\boldsymbol{v}}(t))$ and $(\boldsymbol{c}(t), \boldsymbol{v}(t))$. Other cases $\left(\left(\delta_{i}(C), \delta_{i+1}(C)\right)=\right.$ $(-1,+1),(+1,+1),(-1,-1))$ are similar. This completes the proof of Theorem 5.1 , 


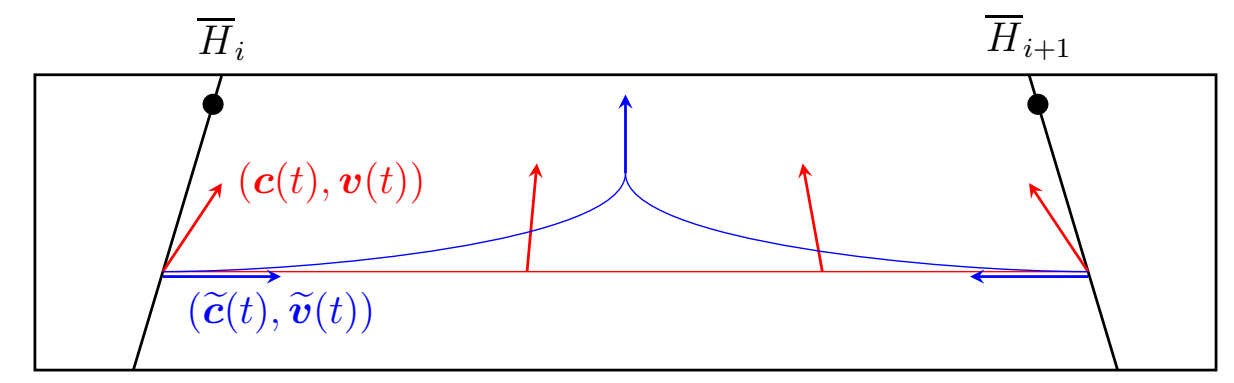

Figure 23: Isotopy between $(\widetilde{\boldsymbol{c}}(t), \widetilde{\boldsymbol{v}}(t))$ and $(\boldsymbol{c}(t), \boldsymbol{v}(t))$

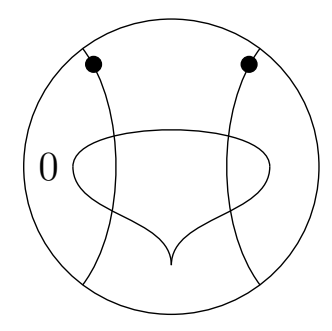

Figure 24: A divide with a cusp whose link is the Kirby diagram of the minimal handle decomposition of $\Sigma_{0,2} \times \Sigma_{0,2}$

\subsection{Further examples}

Let $\Sigma_{g, n}$ be the oriented surface of genus $g$ with $n$ boundary components. Since $\Sigma_{0,2}$ is the annulus, the interior of $\Sigma_{0,2} \times \Sigma_{0,2}$ is homeomorphic to $\left(\mathbb{C}^{\times}\right)^{2}$ which is the complement of the arrangement of two lines intersecting at one point. Hence, the minimal handle decomposition of $\Sigma_{0,2} \times \Sigma_{0,2}$ can be expressed in terms of divide with cusps (Figure 24). The below are related examples.

Example 5.5. The space $\Sigma_{0,4} \times \Sigma_{0,2}$ is realized as the complexified complement of different arrangements $\mathcal{A}_{1}=\left\{\left\{x_{1}=0\right\},\left\{x_{2}=0\right\},\left\{x_{1}=x_{2}\right\},\left\{x_{1}=-x_{2}\right\}\right\}$, and $\mathcal{A}_{2}=\left\{\left\{x_{1}=0\right\},\left\{x_{2}=\right.\right.$ $\left.0\},\left\{x_{2}=1\right\},\left\{x_{2}=2\right\}\right\}$. (Figure 25)
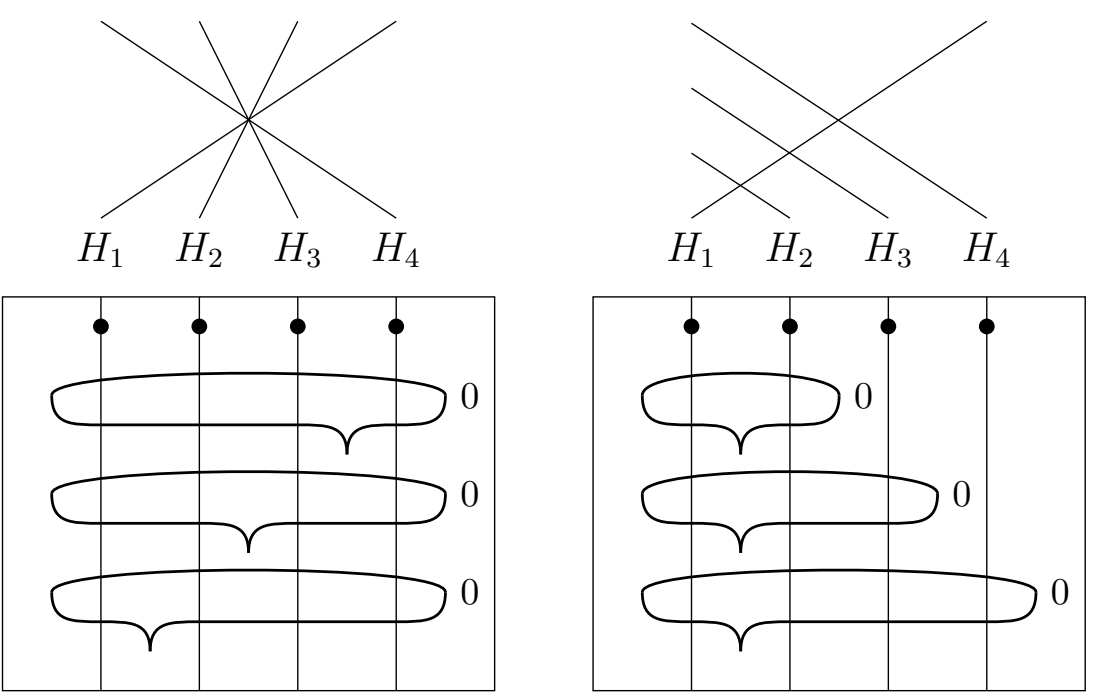

Figure 25: Different divides with cusps for $\Sigma_{0,4} \times \Sigma_{0,2}$

Example 5.6. The space $\Sigma_{0,4} \times \Sigma_{0,3}$ is realized as the complexified complement of the arrangement $\mathcal{A}=\left\{\left\{x_{1}=0\right\},\left\{x_{1}=1\right\},\left\{x_{2}=0\right\},\left\{x_{2}=1\right\},\left\{x_{2}=2\right\}\right\}$. (Figure 26)

Acknowledgements. Masahiko Yoshinaga was partially supported by JSPS KAKENHI Grant Numbers JP19K21826, JP18H01115. The authors thank Prof. Masaharu Ishikawa for helpful 


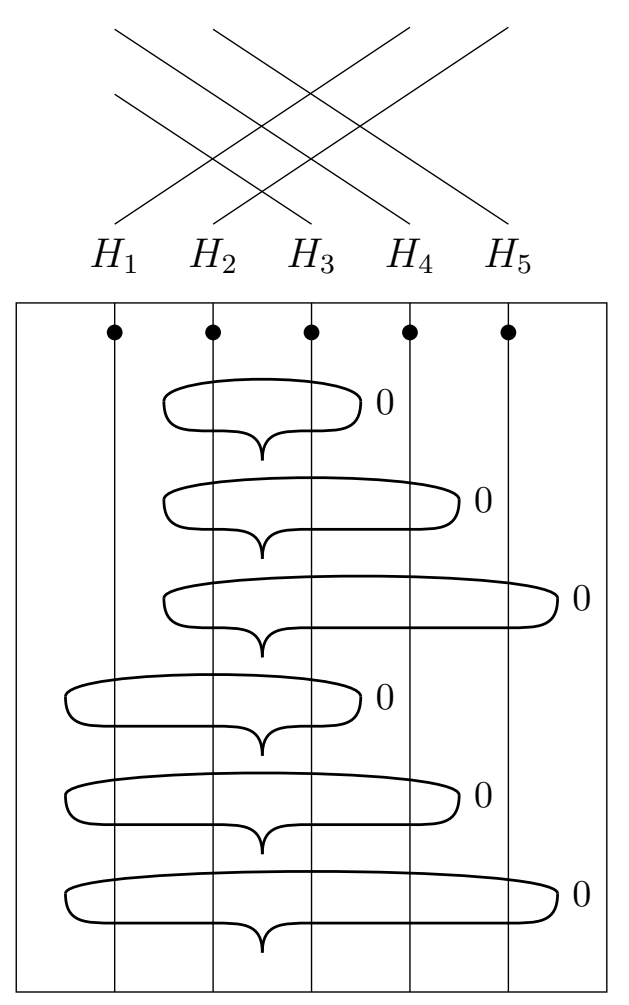

Figure 26: A divide with cusps for $\Sigma_{0,4} \times \Sigma_{0,3}$

comments on the first draft. They also thank the referee for careful reading and comments to improve the paper.

\section{References}

[AC98] N. A'Campo, Generic immersions of curves, knots, monodromy and Gordian number. Inst. Hautes Études Sci. Publ. Math. No. 88 (1998), 151-169.

[Ak16] S. Akbulut, 4-manifolds. Oxford Graduate Texts in Mathematics, 25. Oxford University Press, Oxford, 2016. xii+262 pp.

[CM98] J. A. Calvo, K. C. Millett, Minimal edge piecewise linear knots. Ideal knots, 107-128, Ser. Knots Everything, 19, World Sci. Publ., River Edge, NJ, 1998.

[CP00] O. Couture, B. Perron, Representative braids for links associated to plane immersed curves. J. Knot Theory Ramifications 9 (2000), no. 1, 1-30.

[DP03] A. Dimca, S. Papadima, Hypersurface complements, Milnor fibers and higher homotopy groups of arrangements. Ann. of Math. (2) 158 (2003), no. 2, 473-507.

[Dur83] A. H. Durfee, Neighborhoods of algebraic sets. Trans. Amer. Math. Soc. 276 (1983), no. $2,517-530$.

[F93] M. Falk, Homotopy types of line arrangements. Invent. Math. 111 (1993), no. 1, 139-150.

[GI02a] W. Gibson, M. Ishikawa, Links and Gordian numbers associated with generic immersions of intervals. Topology Appl. 123 (2002), no. 3, 609-636.

[GI02b] W. Gibson, M. Ishikawa, Links of oriented divides and fibrations in link exteriors. Osaka J. Math. 39 (2002), no. 3, 681-703. 
[GS99] R. E. Gompf, A. I. Stipsicz, 4-manifolds and Kirby calculus. Graduate Studies in Mathematics, 20. American Mathematical Society, Providence, RI, 1999. xvi+558 pp.

[IO20] G. Ishikawa, M. Oyama, Topology of complements to real affine space line arrangements. J. Singul. 22 (2020), 373-384.

[K89] R. Kirby, The topology of 4-manifolds. Lecture Notes in Mathematics, 1374. SpringerVerlag, Berlin, 1989. vi+108 pp.

[OT92] P. Orlik, H. Terao, Arrangements of Hyperplanes. Grundlehren Math. Wiss. 300, Springer-Verlag, New York, 1992.

[R02] R. Randell, Morse theory, Milnor fibers and minimality of hyperplane arrangements. Proc. Amer. Math. Soc. 130 (2002), no. 9, 2737-2743.

[SS07] M. Salvetti, S. Settepanella, Combinatorial Morse theory and minimality of hyperplane arrangements. Geom. Topol. 11 (2007), 1733-1766.

[Y07] M. Yoshinaga, Hyperplane arrangements and Lefschetz's hyperplane section theorem. Kodai Math. J. 30 (2007) no. 2, 157-194.

[Y12] M. Yoshinaga, Minimal stratifications for line arrangements and positive homogeneous presentations for fundamental groups. Configuration Spaces: Geometry, Combinatorics and Topology, 503-533, CRM Series, 14, Ed. Norm., Pisa, 2012. 